INTERVIEW

\title{
Biosims séduit Oséo avec ses biopuces
}

\author{
_Propos recueillis par Ludovic Dupin
}

Le concours 2010 Oséo-ministère de la Recherche a récompensé, dans la catégorie création d'entreprise, la start-up Biosims (www.biosims.fr). Créée début novembre 2010, elle est en cours de développement dans la pépinière Seine Biopolis à Rouen. Biosims développe une technologie de biopuces lues par spectromètre SIMS (Secondary Ions Mass Spectrometry) afin d'identifier des marqueurs protéiques. Les deux fondateurs en sont Christine Houclin, présidente, et Guillaume Legent, directeur scientifique.

Biotribune : Quel est le projet développé par Biosims?

Christine Houclin : Chez Biosims, nous développons, avant tout, une technologie de biopuces lues par spectrométrie. Nous voulons que cette application devienne, à terme, l'un des standards de la lecture et de l'analyse clinique. Concrètement, nous avons orienté notre technologie de biopuces sur l'analyse des protéines. En effet, les biopuces à fluorescence fonctionnent très bien pour l'ADN car cette molécule géante peut être amplifiée. Mais, les protéines ne peuvent pas subir le même traitement, aussi il y a de réels problèmes de sensibilité et de quantification avec la fluorescence. La mesure des protéines nous apparaît essentielles en diagnostic car la protéine représente l'ultime expression génétique. En mesurant une protéine anormale, nous sommes certains de mesurer une donnée fiable. De son côté, un gène peut ne pas être exprimé.

BT : Quels types d'applications visez-vous? $\mathrm{CH}$ : Notre berceau a orienté nos premiers domaines d'application. Les équipes de I'Université de Rouen sont originales. II y a d'un côté des bio-physico-chimistes, qui sont des spécialistes des SIMS et de leur application à la biologie. Et de l'autre, il y a des praticiens hospitaliers qui travaillent sur les leucémies et les leucémies infantiles. En croisant les idées, nous avons travaillé sur des premières biopuces pour des applications de dépistage de cancers et de leucémies. Nous disposons d'autres applications potentielles comme les maladies neurodégénératives sur lesquelles nous avons déjà travaillé avec des partenaires industriels.

Théoriquement, notre champ d'application est très large. Ce sont les cahiers des charges de nos clients industriels qui vont définir les évolutions de notre technologie. La seule limitation de notre innovation est de disposer de l'anticorps correspondant à la protéine recherchée.

BT : Quel est l'intérêt de votre technologie par rapport à d'autres alternatives en cours de développement?

CH : La simplicité. La détection des protéines est souvent lourde et peu sensible. Pour notre part, en nous focalisant à terme sur les applications cliniques, il y aura d'un côté un appareil de lecture simple d'usage et de l'autre côté il y aura des biopuces caractérisées par rapport à un diagnostic de maladie. Ces puces, en définitive, seront presque "pré-interprétées ».

BT : A quel stade de développement en est votre technologie?

CH: Aujourd'hui, notre technologie est encore à un stade préindustriel. Elle peut d'ores et déjà être utilisée pour faire des études de recherche de biomarqueurs. Mais nous ne pouvons pas encore vendre de lecteurs dans les hôpitaux, afin que ces derniers réalisent leurs tests eux-mêmes.

BT : Quel est l'intérêt pour Biosims d'être lauréat du concours Oséo-ministère de la Recherche?

CH : Ce concours est important sur deux points. II y a d'abord l'aspect financier pour lequel le prix Oséo est un amorçage important. II est la première brique qui permet de lever les autres sources de financement comme les Jeunes Entreprises Innovantes et le Crédit Impôt Recherche. Cette source financière nous permettra d'embaucher deux docteurs, dès janvier. Le premier travaillera sur l'industrialisation de la technologie et le second va s'atteler au développement d'un premier catalogue d'applications sous forme de prestations de service. Le deuxième point est bien sût l'aspect « label » qui nous ouvre énormément de portes.

BT : Quel est votre modèle économique? CH : Pour l'heure, nous sommes prestataires de services. Nous nous adressons à des organismes de recherches académiques ou industriels. Que ces derniers travaillent sur de nouveaux traitements et souhaitent suivre les marqueurs compagnons de ce traitement, ou qu'ils s'intéressent directement à des problématiques de diagnostic. Nous espérons lancer les premières opérations commerciales au premier trimestre 2011.

Dans un second temps, nous souhaitons développer un outil de lecture de ces biopuces, très vraisemblablement commercialisé et fabriqué par des industriels partenaires. Biosims, de son côté, développera et commercialisera les biopuces. Nous avons déjà plusieurs contacts industriels et nous effectuons une sélection basée sur des critères technologiques et économiques.

BT : Que vous manque-t-il pour franchir les étapes suivantes?

CH : Aujourd'hui, il nous manque des applications. Notre technologie fonctionne sur des modèles de laboratoire. Mais elle n'a jamais été mise en œuvre lors d'une étude préclinique. Nous serions intéressés par un rapprochement avec une équipe prête à collaborer pour obtenir une preuve de concept. Dans l'idéal, nous souhaiterions réaliser cette opération en cancérologie, de préférence dans des domaines où les marqueurs sont en très faibles quantités. 